\title{
Nondestructive Evaluation of Concrete within Nuclear Applications
}

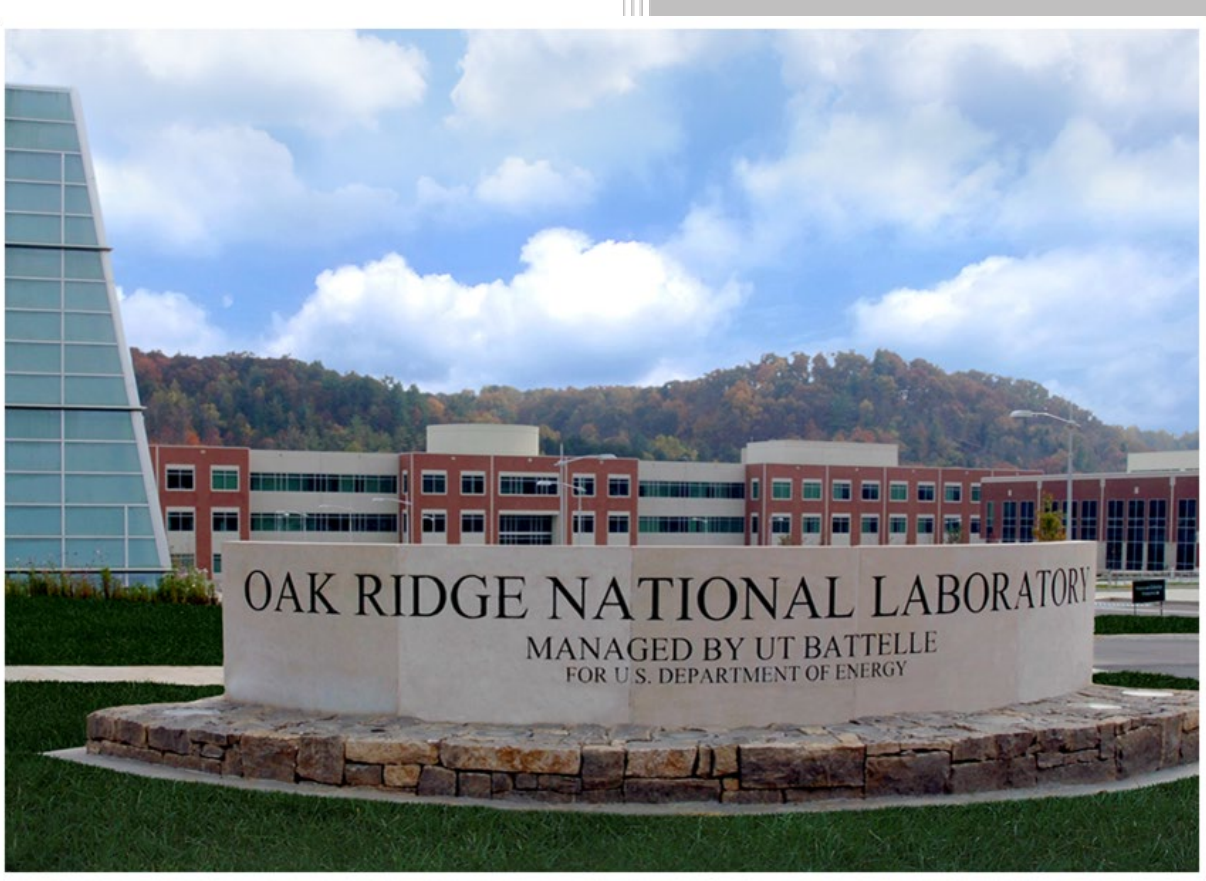

Approved for public release. Distribution is unlimited

N. Dianne Bull Ezell

August 2020 


\section{DOCUMENT AVAILABILITY}

Reports produced after January 1, 1996, are generally available free via US Department of Energy (DOE) SciTech Connect.

Website http://www.osti.gov/scitech/

Reports produced before January 1, 1996, may be purchased by members of the public from the following source:

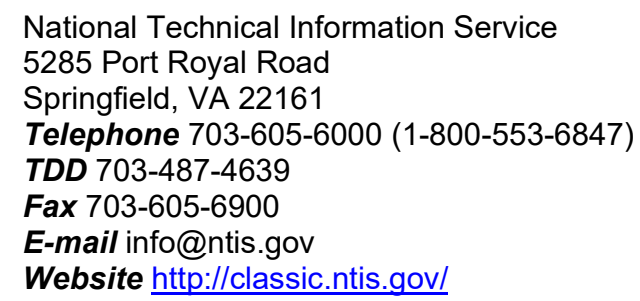

Reports are available to DOE employees, DOE contractors, Energy Technology Data Exchange representatives, and International Nuclear Information System representatives from the following source:

Office of Scientific and Technical Information

PO Box 62

Oak Ridge, TN 37831

Telephone 865-576-8401

Fax 865-576-5728

E-mail reports@osti.gov

Website http://www.osti.gov/contact.html

This report was prepared as an account of work sponsored by an agency of the United States Government. Neither the United States Government nor any agency thereof, nor any of their employees, makes any warranty, express or implied, or assumes any legal liability or responsibility for the accuracy, completeness, or usefulness of any information, apparatus, product, or process disclosed, or represents that its use would not infringe privately owned rights. Reference herein to any specific commercial product, process, or service by trade name, trademark, manufacturer, or otherwise, does not necessarily constitute or imply its endorsement, recommendation, or favoring by the United States Government or any agency thereof. The views and opinions of authors expressed herein do not necessarily state or reflect those of the United States Government or any agency thereof. 
Reactor and Nuclear Systems Division

\section{Nondestructive Evaluation of Concrete within Nuclear Applications}

N. Dianne Bull Ezell

Date Published: August 2020

Prepared by

OAK RIDGE NATIONAL LABORATORY

Oak Ridge, TN 37831-6283

managed by

UT-BATTELLE, LLC

for the

US DEPARTMENT OF ENERGY

under contract DE-AC05-00OR22725 



\section{CONTENTS}

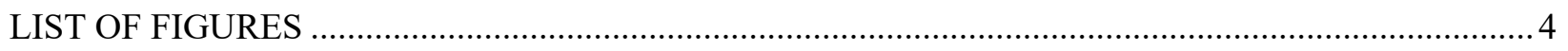

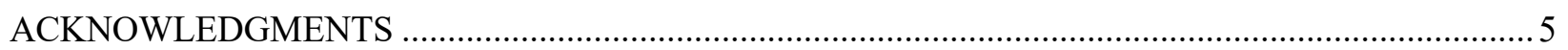

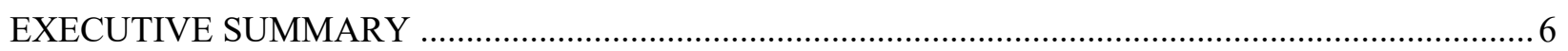

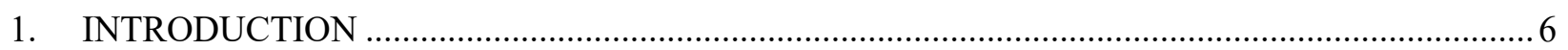

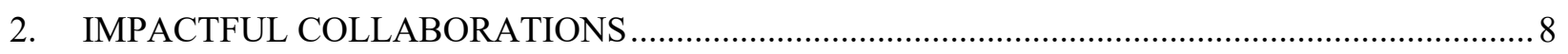

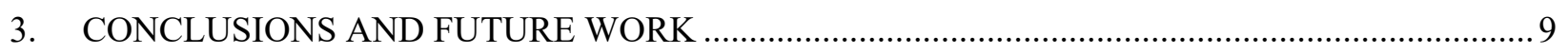

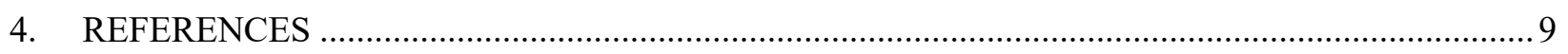




\section{LIST OF FIGURES}

Figure 1. Illustration of a frequency synthesis-focusing FB-SAFT technique. …................................. 7

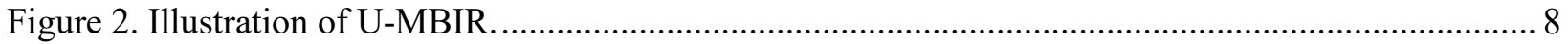




\section{ACKNOWLEDGMENTS}

This work is funded by the US Department of Energy's Office of Nuclear Energy under the Light Water Reactor Sustainability program. 


\section{EXECUTIVE SUMMARY}

Structural defects that develop over the lifetime of nuclear power plants (NPPs) could threaten the safety and security of the concrete infrastructure. Developing a reliable nondestructive evaluation (NDE) method to evaluate the damage in NPPs and their associated structures will greatly improve the long-term operation and safety of NPPs. The purpose of the US Department of Energy Office of Nuclear Energy's Light Water Reactor Sustainability program is to develop technologies and other solutions that can improve the reliability, sustain the safety, and extend the operating lifetimes of NPPs beyond 60 years. This report describes the progress made toward growing NDE capabilities for NPPs. It also documents expanded collaborations with the Electric Power Research Institute (EPRI) and future opportunities to collaborate with industry partners (i.e., vendors and utilities) and university partners. These collaborations will continue to stand as a framework to provide value to stakeholders by reducing uncertainties and potential delayed maintenance costs in NPPs.

\section{INTRODUCTION}

Structural defects that develop over the lifetime of nuclear power plants (NPPs) could threaten the safety and security of the concrete infrastructure. These defects form due to natural aging, environmental impacts, or flawed construction. The harsh nuclear environment adds significant difficulty to the detection and correction of concrete defects because often times this means sending individuals into radiological areas. Coring and other destructive testing methods are typically employed, but this is not an ideal structural health-monitoring solution for NPPs because it introduces fatigue to the infrastructure. Developing a reliable nondestructive evaluation (NDE) method to evaluate the damage in these structures will greatly improve the long-term operation and safety of NPPs.

Oak Ridge National Laboratory (ORNL) has supported the NDE of concrete under the Light Water Reactor Sustainability (LWRS) program [1-7]. This effort has included the development of concrete specimens to study the detection of known concrete defects, such as delamination, voids, honeycombing, and alkali-silica reaction (ASR). Several of these studies evaluated increasingly complex and thicker concrete specimens. Initial efforts investigated methods and instrumentation that are commercially available for NDE, as well as performed research and development on new instrumentation [8].

This report describes the progress and growth made toward growing NDE capabilities. Despite the limited budget and restricted travel, this task continued to contribute to the NDE community through conferences, such as SPIE Smart Structures and NDE and quantitative NDE, and through digital meetings, such as Electric Power Research Institute (EPRI) roadmap meetings, LWRS program reviews, and collaborative discussion. The task also focused on supporting and leading several US Department of Energy Office of Nuclear Energy proposals that address structural health monitoring and NDE with industry and university partners.

Collaborations with university partners shifted the focus of these studies from developing the physical instrumentation to creating reconstruction algorithms that are used to generate multi-dimensional images from the data acquired by the NDE measurement to maximizing the information. Applying advanced image reconstruction algorithms to the instrumentation enables a more complete analysis while on-site. Typically, ultrasonic instrumentation uses a synthetic aperture focusing technique (SAFT) for real-time image reconstructions. SAFT reconstructions generate a cross sectional image by using a specialized Bscan, which is a compilation of many A-scans (i.e., the output of an ultrasonic sensor). It is a delay-andsum technique that uses a time-of-flight signal and the speed of sound in the medium. A color-based map is created by using the signal amplitude. Converting time to distance by using the propagation velocity of the material enables the calculation of the round-trip distance for each transmit and receive sensor pair. 
However, these reconstructions contain artifacts and background noise that disguise and obscure defects that may be present. To address these issues, research focused on the development of an improved SAFT algorithm, frequency-banded SAFT (FB-SAFT) [9], that uses frequency banding of the B-scans post SAFT processing to reduce noise and artifacts in the images, as shown in Figure 1. This image reconstruction technique is more complex than standard SAFTs but only requires a small addition of computational time.

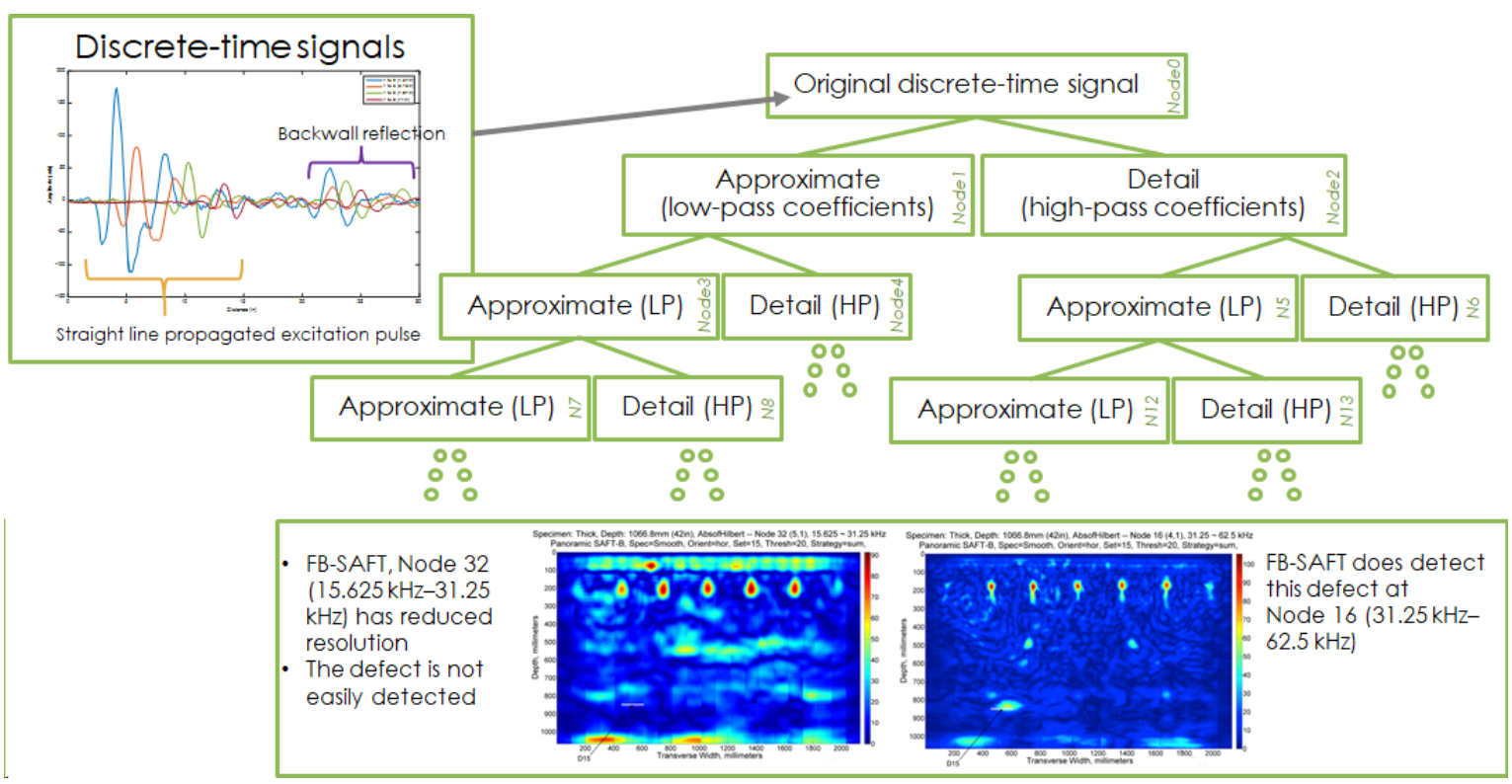

Figure 1. Illustration the FB-SAFT technique. The discrete-time signals are filtered through the use of a wavelet packet decomposition followed by selective reconstruction based on the retention of desired frequency bands. Node

$16(31.25-62.5 \mathrm{kHz})$, right sub-image, is typically the best reconstruction band as the center frequency of the instrument used to collect this data falls inside the $31.25-62.5 \mathrm{kHz}$ band. In Node $32(15.625-31.25 \mathrm{kHz})$, the defect is obscured due to the additional, but uninformative, energy in that band.

Although the FB-SAFT reconstructions greatly improved the SAFT reconstructions, the method limits the data obtained from the entire measurement bandwidth. It also limits the signal of interest, even though it limits artifacts and background noise. To address the loss of data, an ultrasonic model-based iterative reconstruction (U-MBIR) algorithm was developed. A typical U-MBIR problem contains two main parts: the forward model that describes the physical system (i.e., measurements) and the prior model that describes the object (i.e., image) to be reconstructed, as shown in Figure 2. Prior models are based on known image reconstruction techniques, such as edge detection, physical medium properties, and shadowing due to solids in the medium. This method is more computationally time consuming due to the iterative steps but is also more powerful in image reconstructions and is different from FB-SAFT because it uses the entire bandwidth of data. The U-MBIR method requires more research to decrease the processing time from several hours to several minutes; however, a clear path for accomplishing this was identified, which included using SAFT reconstructions as a prior (i.e., image) model. Both reconstruction techniques are discussed in greater detail in multiple reports and publications [1-5]. 

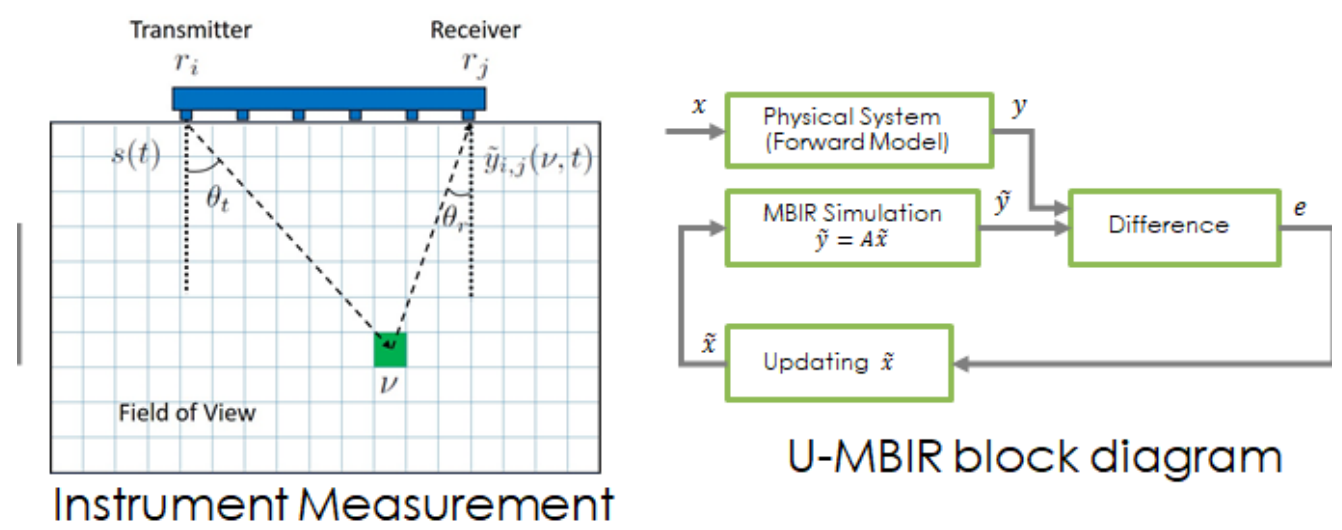

Figure 2. Illustration of U-MBIR.

\section{IMPACTFUL COLLABORATIONS}

Understanding structural health monitoring requires a diverse research team including civil engineers, electrical engineers, nuclear engineers, and material scientist. Through the LWRS Program, ORNL's concrete NDE team has collaborated with EPRI for several years, combining basic science expertise and field experience. EPRI certifies and creates field guides, such as the roadmaps built within their research programs, based on this collaborative research. The goal behind the concrete NDE roadmap development is to identify gap areas and prevent the duplication of research efforts, which dilutes research funding. The concrete NDE roadmap is divided into three research areas including physical NDE, approach and degradation mechanisms, and structural health monitoring. Tasks were planned through the year 2023 for ORNL and INL, university partners, EPRI, industry, and US Nuclear Regulatory Commission participants. Contributors are expected to bring their own funding but contribute equally to the development of this high-impact program.

Areas of collaboration were identified through the roadmap development. For example, EPRI is very interested in applying the LWRS U-MBIR method to the ultrasonic tomography systems at its concrete demonstration facility. The ultrasonic tomography system will process the data by using internal software - likely a SAFT reconstruction - to produce initial reconstructions. Then, U-MBIR would postprocess the data to allow additional information to be obtained from the original dataset. EPRI would then provide feedback to ORNL about possible improvements that could lead to software commercialization. ORNL would provide this tool to EPRI for free. However, end users must be trained to use the tool, so funding is needed to pay for training hours.

EPRI has developed an extensive concrete laboratory at its Charlotte, North Carolina office that includes a large-scale experiment that was recently designed to contain intentionally introduced, well-known concrete defects in nine different specimens. These large rectangular slabs were created to allow collaborators to establish the limitations of their equipment (e.g., depth of measurement, edge effects, ability to see around solids), demonstrate the capability to detect defects (e.g., minimal size of defects), and further develop equipment and data analysis algorithms. Furthermore, an additional set of nine mockups with similar characteristics were fabricated in Europe to allow for international collaboration. To enable experimenters to demonstrate their defect detection capability, the information regarding the location and makeup of the defects is withheld so that the experiment is considered blind. The participants report their results, which are compared with the as-built characteristics of the mockups. The concrete NDE team used ultrasonic measurements collected on the concrete specimens to continue development on the data analysis algorithms. This year, negotiations on intellectual property were discussed to create a 
collaborative agreement between ORNL and EPRI that addresses this experiment. Additionally, EPRI requested control of the publication of these results until all measurements are completed and the results are reported to EPRI.

Potential collaborations with industry partners were initiated, with one vendor requesting ORNL to evaluate NPP NDE and structural health monitoring. This is expected to be a multiyear collaboration with several other collaborators, including EPRI. ORNL will leverage extensive instrumentation experience to identify existing solutions and develop novel techniques by using embedded sensors for material studies and demonstrations. Moreover, this research would build on the fiber-optic research developed under the LWRS NDE and structural health monitoring of large concrete specimens that contain alkali-silica reaction to evaluate concrete degradation, rebar separation, and other longer term NPP failures.

University partnerships that promote more research and development by investigating new techniques and instrumentation within NDE will also be pursued while serving industry in a collaborative role. Based on the experience developed during the ASR evaluations, a consortium is anticipated to be used to coordinate and more effectively disseminate the results for industry use. To date, the ORNL team has worked with the University of Minnesota, Vanderbilt University, the University of Pittsburgh, and the University of Tennessee-Knoxville. The team is also fostering connections between the concrete research team and researchers at the University of Nebraska, Michigan State University, and Iowa State University which are anticipated to lead to the submission of multiple proposals in the coming year. Some proposals will focus on continuing the development of ultrasonic methods and image reconstruction, and others will focus on developing entirely new NDE methods. With this collaboration, the universities would have access to the advanced algorithm development at ORNL and train their students under subject matter experts. Although the algorithms were initially developed for application to ultrasonic measurements, the research team thinks that the algorithms can be modified for application to other instrumentation.

Partnerships with universities that are developing novel instrumentation would create opportunities for expanded algorithm applications. This is expected to reduce the costs of developing these instruments and provide image reconstruction options for the universities. Based on this initial work, the ORNL NDE team was invited to serve on the Industry Advisory Board within the Iowa State University's Center for Nondestructive Evaluation.

\section{CONCLUSIONS AND FUTURE WORK}

This report documents the expanded collaborations with EPRI and opportunities with industry (i.e., vendors and utilities) and university partners. Although the limited FY20 funding and travel restrictions directly impacted the pace of building the collaborations, it provided the framework for providing value to stakeholders by reducing uncertainties and potential maintenance costs.

\section{REFERENCES}

1. Ezell, N. D. B., Albright, A., Floyd, D., Clayton, D., and Khazanovich, L. "A Novel Use of Frequency-Banded Synthetic Aperture Focusing Technique for Reconstructions of Alkali-Silica Reaction in Thick-Reinforced Concrete Structures." AIP Conference Proceedings 2102, no. 1 (2019). AIP Publishing.

2. Ezell, N. Dianne Bull, Venkatakrishnan, S. V., Al Mansouri, H., Santos-Villalobos, H., and Floyd, D. "High Fidelity Ultrasound Imaging of Concrete Structures." Smart Structures and NDE for Energy Systems and Industry 4.0. 10973 (2019). International Society for Optics and Photonics.

3. Ezell, N. D. B., Albright, A., Clayton, D., and Santos-Villalobos, H. "Detecting Alkali-Silica Reaction in Thick Concrete Structures Using Linear Array Ultrasound." Nondestructive 
Characterization and Monitoring of Advanced Materials, Aerospace, Civil Infrastructure, and Transportation XII 10599 (2018). International Society for Optics and Photonics.

4. Ezell, N. D. B., Hayes, N., Lenarduzzi, R., Clayton, D., Ma, Z. J., Le Pape, S., and Le Pape, Y. "Experimental Collaboration for Thick Concrete Structures with Alkali-Silica Reaction." AIP Conference Proceedings 1949, no. 1 (2018). AIP Publishing.

5. Ezell, N D. Bull, Venkatakrishnan, S.V., Santos-Villalobos, H., Albright, A. Comparative Analysis of Nondestructive Examination Techniques of Enhanced Model Based Iterative Reconstruction (MBIR) and Frequency-banded Synthetic Aperture Focusing Technique (SAFT) Reconstructions. ORNL/SPR2019/1240. Oak Ridge National Laboratory, Oak Ridge, Tennessee (2019).

6. Clayton, D. A. and Hileman, M. S. Light Water Reactor Sustainability Non-Destructive Evaluation for Concrete Research and Development Roadmap. ORNL/TM-2012/360, Oak Ridge National Laboratory, Oak Ridge, Tennessee (2012).

7. Smith, C. et al. Roadmap for Nondestructive Evaluation of Reactor Pressure Vessel Research and Development by the Light Water Reactor Sustainability Program. ORNL/TM-2012/380, Oak Ridge National Laboratory, Oak Ridge, Tennessee (2012).

8. Clayton, D. A. et al. "Research and Development Roadmaps for Nondestructive Evaluation of Cables, Concrete, Reactor Pressure Vessels, and Piping Fatigue." Nondestructive Characterization for Composite Materials, Aerospace Engineering, Civil Infrastructure, and Homeland Security 8694 (2013). International Society for Optics and Photonics.

9. Albright, A., Clayton, D. "Improved Synthetic Aperture Focusing Technique Results of Thick Concrete Specimens through Frequency Banding” AIP Conference Proceedings 1706, no. 1 (2014). AIP Publishing. 